\title{
Colistin Resistance of Pseudomonas aeruginosa Isolated from Snakes in Taiwan
}

\author{
Po-Yu Liu, ${ }^{1,2}$ Ling-Ling Weng, ${ }^{3}$ Shu-Ying Tseng, ${ }^{3}$ Chou-Chen Huang, \\ Ching-Chang Cheng, ${ }^{5}$ Yan-Chiao Mao, ${ }^{6}$ and Kwong-Chung Tung ${ }^{3}$ \\ ${ }^{1}$ Department of Internal Medicine, Taichung Veterans General Hospital, Taichung, Taiwan \\ ${ }^{2}$ Rong Hsing Research Center for Translational Medicine, National Chung Hsing University, Taichung, Taiwan \\ ${ }^{3}$ Department of Veterinary Medicine, College of Veterinary Medicine, National Chung Hsing University, Taichung, Taiwan \\ ${ }^{4}$ Veterinary Teaching Hospital, College of Veterinary Medicine, National Chiayi University, Chiayi City, Taiwan \\ ${ }^{5}$ Laboratory Animal Service Center, Office of Research and Development, China Medical University, Taichung 40402, Taiwan \\ ${ }^{6}$ Department of Emergency Medicine, Taichung Veterans General Hospital, Taichung 40402, Taiwan
}

Correspondence should be addressed to Kwong-Chung Tung; kctung98@gmail.com

Received 9 May 2017; Accepted 17 August 2017; Published 25 September 2017

Academic Editor: Branka Bedenić

Copyright (C) 2017 Po-Yu Liu et al. This is an open access article distributed under the Creative Commons Attribution License, which permits unrestricted use, distribution, and reproduction in any medium, provided the original work is properly cited.

\begin{abstract}
This study included fifty-eight isolates of $P$. aeruginosa from the oral cavity of snakes that were recruited from clinical cases, captive and wild snakes. The minimum inhibitory concentrations (MICs) for the determination of susceptibility were identified by the broth microdilution method. Polymerase chain reaction (PCR) was employed to detect $\beta$-lactamases genes. With regard to antipseudomonal antibiotics, the lowest nonsusceptible rates were in aztreonam (15\%), piperacillin/tazobactam (12\%), and amikacin (9\%). The nonsusceptible rates were high in gentamicin (33\%) and colistin (55\%). Meanwhile, $b l a_{\text {TEM }}$ presented in $100 \%$ of isolates where $b l a_{\mathrm{AmpC}}, b l a_{\mathrm{OXA}-1}$, and $b l a_{\mathrm{OXA}-10}$ came at $94.8 \%, 89.7 \%$, and $27.6 \%$, respectively. Emergence of multidrug resistant (MDR) strains and colistin-resistant strains highlights the potential breach of public health as $P$. aeruginosa could be transmitted through either direct contact or indirect dissemination through the environment. This study reports that the highly resistant $P$. aeruginosa from snakes' oral cavity were discovered for the very first time in Taiwan.
\end{abstract}

\section{Introduction}

Antibiotic resistance poses a growing threat to public health all over the globe. Undoubtedly inappropriate prescription is a major issue, and that in agriculture and husbandry deserves more focus. Moreover, surging level of antimicrobial agents employed by veterinary medicine makes animals today potential reservoirs of resistant bacteria [1].

Categorized as opportunistic pathogen, nonetheless, Pseudomonas aeruginosa infection could be lethal in the immunocompromised population [2]. Yet the pivotal problem lies in the intrinsic antibiotic resistance of $P$. aeruginosa that counteracts the effects of medical treatments [3]. To current knowledge, $P$. aeruginosa is more likely to be an environmental species and passed on by animal reservoirs, the snakes, for instance [3]. In fact, the latest report from Taiwanese Centers of Disease Control illustrates that incidence of snakebites reaches more than 1,000 cases annually [4] and implicates underestimated role of $P$. aeruginosa of snake origin.

However, little is detailed about the determination regarding susceptibility of antibiotics that are widely administered within human population such as piperacillin/tazobactam and meropenem [5]. Since disease-free snakes might be carriers as well, captive ones of commercial origin and from research institution were also enrolled in our study. In brief, sensitivity testing of $P$. aeruginosa from snakes' oral cavities and subsequent molecular typing of resistance genes will be outlined in the following paragraph, with further implication of clinical translation and public health responses.

\section{Materials and Methods}

2.1. Pseudomonas aeruginosa Strains. The study was implemented based on 58 P. aeruginosa isolates from disease-free 
TABLE 1: Primers sequences used in the amplification and antimicrobial resistance genes in P. aeruginosa.

\begin{tabular}{lcccc}
\hline Class of $\beta$-lactamases & Primer name & Sequence $\left(5^{\prime}\right.$ to $\left.3^{\prime}\right)$ & Product size (bp) & Target \\
\hline \multirow{2}{*}{ Class A } & TEM-A & GAGTATTCAACATTTCCGTGTC & 851 & \\
& TEM-B & TAATCAGTGAGGCACCTATCTC & 760 & blaTEM \\
& OXA-10F & TCTTTCGAGTACGGCATTAGC & OXA group I \\
Class D & OXA-10B & CCAATGATGCCCTCACTTTCC & OXA group III \\
& OXA-1A & AGCCGTTAAAATTAAGCCC & 1243 \\
OXA-1B & CTTGATTGAAGGGTTGGGCG & blaAmpC \\
\hline
\end{tabular}

TABLE 2: Distribution of MICs and antimicrobial sensitivity test of gentamicin, amikacin, cefotaxime, piperacillin/tazobactam, colistin, meropenem, and aztreonam for $P$. aeruginosa.

\begin{tabular}{|c|c|c|c|c|c|c|c|c|c|c|c|c|c|c|c|}
\hline \multirow{2}{*}{ Agent } & \multicolumn{12}{|c|}{ Number of isolates with indicated MIC values $(\mathrm{mg} / \mathrm{L})$} & \multicolumn{3}{|c|}{ Percentage of indicated susceptibility } \\
\hline & 0.125 & 0.25 & 0.5 & 1 & 2 & 4 & 8 & 16 & 32 & 64 & 128 & $\geq 256$ & $S(\%)$ & $I(\%)$ & $R(\%)$ \\
\hline Gentamicin & 0 & 0 & 0 & 0 & 5 & 34 & 11 & 2 & 0 & 0 & 1 & 5 & $39(67 \%)$ & $11(19 \%)$ & $8(14 \%)$ \\
\hline Amikacin & 0 & 0 & 0 & 0 & 0 & 28 & 19 & 6 & 0 & 0 & 0 & 5 & $53(91 \%)$ & 0 & $5(9 \%)$ \\
\hline Cefotaxime & 0 & 0 & 0 & 0 & 0 & 2 & 4 & 18 & 17 & 9 & 6 & 2 & $6(10 \%)$ & $18(31 \%)$ & 34 (59\%) \\
\hline Pip + taz & 0 & 0 & 0 & 0 & 0 & 14 & 28 & 9 & 5 & 2 & 0 & 0 & $51(88 \%)$ & $7(12 \%)$ & 0 \\
\hline Colistin & 0 & 0 & 0 & 0 & 26 & 25 & 2 & 0 & 2 & 0 & 0 & 3 & $26(45 \%)$ & $25(43 \%)$ & $7(12 \%)$ \\
\hline Meropenem & 6 & 18 & 8 & 17 & 8 & 1 & 0 & 0 & 0 & 0 & 0 & 0 & 57 (98\%) & $1(2 \%)$ & 0 \\
\hline Aztreonam & 0 & 0 & 0 & 0 & 4 & 19 & 26 & 6 & 2 & 0 & 0 & 1 & $49(85 \%)$ & $6(10 \%)$ & $3(5 \%)$ \\
\hline
\end{tabular}

Pip + taz: piperacillin/tazobactam; $S$ : susceptible; $I$ : intermediate; $R$ : resistant.

captivated snakes, wild snakes at Endemic Species Research Institute (ESRI), and clinical cases at National Chung Hsing University Veterinary Medicine Teaching Hospital, Taichung, Taiwan. All samples were collected through sterilized swabbing and stored in the transport media on the way to microbiology laboratory. Following aerobic culture routine, the isolates were plated onto cetrimide agar and incubated at $35^{\circ} \mathrm{C}$ in $5 \% \mathrm{CO} 2$ and $95 \%$ air for 48 hours. Based on the acquired colonies, Pseudomonas aeruginosa was further determined by colony morphology, Gram stain, oxidase test, and API ID 32 GN strips (bioMérieux, Marcy l'Etoile, France).

2.2. Antimicrobial Susceptibility Testing. The minimum inhibitory concentrations (MICs) were identified by Clinical Laboratory Standards Institute (CLSI) [6]. Targeting $P$. aeruginosa, cation-adjusted Müller-Hinton broth was engaged to subsequent serial twofold dilutions of representative antimicrobial agents. $P$. aeruginosa suspension was then adjusted to the turbidity of $0.5 \mathrm{McFarland}$ standard of $P$. aeruginosa ATCC27853, and $100 \mu \mathrm{L}$ of the bacteria with inoculum density at around $10^{5} \mathrm{CFU} / \mathrm{mL}$ was further transferred onto 96-well plates. After incubation at $37^{\circ} \mathrm{C}$ for 24 hours, MICs of respective antibiotics were then categorized into susceptible, intermediate, and resistant based on the interpretation table within CLSI guidance. The antimicrobials were employed in the study: penicillins (piperacillin/tazobactam), cephems (cefotaxime), aminoglycosides (gentamicin and amikacin), lipopeptides (colistin), monobactams (aztreonam), and carbapenems (meropenem).
2.3. Detection of Resistance Genes in P. aeruginosa Isolates. The Nucleic Acid Automatic Extraction System (Taco, Taichung, Taiwan) was adopted for DNA isolation through the manufacturer's protocol. The extracts served as PCR templates, where primers and the amplification outcomes of bla $a_{\mathrm{TEM}}, b l a_{\mathrm{OXA}}$-group I/III, and $b l a_{\mathrm{AmpC}}$ specific PCR are outlined in Table 1 . In brief, $2 \mu \mathrm{L}$ of DNA template was mixed with $1 \mu \mathrm{L}$ upstream primer, $1 \mu \mathrm{L}$ downstream primer, $5 \mu \mathrm{L}$ of Fast-Run ${ }^{\text {TM }}$ Taq Master Mix (Protech Technology Enterprise Co., Ltd., Taipei, Taiwan), and $16 \mu \mathrm{L}$ distilled water to reach $25 \mu \mathrm{L}$ in total. Each premixed set was then anchored within GenePro Thermal Cycler (Bioer Technology, Hangzhou, China). Subsequent to the initial denaturation session of 5 minutes at $95^{\circ} \mathrm{C}$, whole PCR was subjected to 30 cycles of 30 seconds at $94^{\circ} \mathrm{C}$, half an hour at $55-65^{\circ} \mathrm{C}, 45-75$ seconds at $72^{\circ} \mathrm{C}$, and final 7-minute-long extension at $72^{\circ} \mathrm{C}$.

2.4. Statistical Analysis. All data were processed through basic statistic tools of Microsoft Excel (Microsoft Corporation, Redmond, USA).

\section{Results}

3.1. Antimicrobial Resistance. MIC distribution of indicative antimicrobial agents is summarized in Table 2, which illustrates further susceptibility translation according to CLSI standards for broth microdilution method and preexisting references of cefotaxime [6]. Of note, only one out of 58 isolates $(2 \%)$ demonstrated resistance to meropenem otherwise sensitive $(\mathrm{MIC} \leq 2 \mathrm{mg} / \mathrm{L})$. Second to meropenem, 
TABLE 3: Prevalence of Ambler classes A, C, and D $\beta$-lactamases among fifty-eight clinical isolates of P. aeruginosa.

\begin{tabular}{|c|c|c|}
\hline Class & Type of $\beta$-lactamases & Number (\%) of isolates \\
\hline Class A & blaTEM & $58(100 \%)$ \\
\hline \multirow{2}{*}{ Class D } & OXA group I (OXA-1) & $52(89.7 \%)$ \\
\hline & OXA group III (OXA-10) & $16(27.6 \%)$ \\
\hline Class C & blaAmpC & $55(94.8 \%)$ \\
\hline \multirow{5}{*}{ Combined } & blaTEM, OXA-1, OXA-10, blaAmpC & $15(25.9 \%)$ \\
\hline & blaTEM, OXA-1, blaAmpC & $34(58.6 \%)$ \\
\hline & blaTEM, blaAmpC & $5(8.6 \%)$ \\
\hline & blaTEM, OXA-10, blaAmpC & $1(1.7 \%)$ \\
\hline & OXA-1, blaAmpC & $3(5.2 \%)$ \\
\hline
\end{tabular}

approximately $80-90 \%$ of $P$. aeruginosa isolates also exhibited susceptibility to amikacin, piperacillin/tazobactam, and aztreonam. On the opposite, 52 isolates (90\%) were found to be nonsusceptible to cefotaxime, which was followed by colistin at $55 \%$ and gentamicin at $33 \%$ resistance rate, respectively.

3.2. Prevalence of $\beta$-Lactamase Genes. Meanwhile, $\beta$-lactamase gene within the context was identified through PCR and subsequent molecular typing as well. Intriguingly, blaTEM was discovered among all isolates whereas blaAmPC was detected in 55 out of 58 isolates (94.8\%). As for Ambler class $\mathrm{D} \beta$-lactamase family, frequency of OXA-1 was over three times higher than that of OXA-10 (89.7\% over $27.6 \%)$. Remarkably, presence of OXA-10 displayed high correlation (93.8\%) with coexistence of other three resistance genes. Distribution pattern of $\beta$-lactamase genes is abstracted in Table 3.

\section{Discussion}

In our study, antibiotic-resistant $P$. aeruginosa strains from the snakes' oral cavity were identified for the very first time. Of these, resistance rate even reached approximately 50\% with certain antibiotics. High prevalence of $\beta$-lactamases genes was disclosed through our work as well.

In terms of other natural reservoirs, $P$. aeruginosa could only be discovered in a limited number of animals like canine, feline, swan, and reptile [5]. Most studies of animal $P$. aeruginosa strains from Pan-Pacific regions reported relatively subtle resistance [7]. Reportedly, merely $17.8 \%$ of $P$. aeruginosa from dogs and cats demonstrated resistance of cefotaxime whereas over half of reptile strains recruited in our study were insensitive to the chemotherapeutic agents [8]. As for carbapenems, it is rather striking to detect $2 \%$ resistance in reptile strains as their canine and feline parallels were all sensitive according to current literature [8]. Although polymyxin B resistance was once reported in canine $P$. aeruginosa [9], there exists no direct evidence to implicate colistin (also known as polymyxin E) resistance that was expressed by almost $50 \%$ of snake isolates in our study. Combined, to our knowledge, $P$. aeruginosa from snakes' oral cavity are highly antibiotic-resistant.

Indeed $P$. aeruginosa possesses diverse intrinsic resistance mechanisms such as multidrug efflux pumps and antibiotics inactivating enzymes and naturally tolerates a number of antimicrobial agents [10]. Also, the species is likely to develop acquired or adaptive resistance under selective pressure. Although there are certain antipseudomonal antibiotics available, emergence of multidrug resistant (MDR) strains poses a considerable threat to the public health. Since colistin resistance was accidentally discovered through our work, the problem is far more complicated than initially expected.

In Taiwan, MDR P. aeruginosa took up $18.6 \%$ among all hospital-acquired infections in a retrospective review [11], of which the level was similar to that worldwide. A parallel Taiwanese study also illustrated insufficient colistin susceptibility of $P$. aeruginosa, at a mere $76.7 \%$ [12]. Mirrored to our study, colistin resistance of snake $P$. aeruginosa was still $31.7 \%$ higher than that of human strains. On the other hand, MDR reptile strains stood at approximately one-tenth in our study, which was similar to $10-20 \%$ among human $P$. aeruginosa infections in Taiwan [13].

With regard to antibiotic resistance among $P$. aeruginosa in other Asian-Pacific countries, surveillance report in 2011 outlined the highest carbapenem resistance in Philippines (50\%) that was followed by India (32\%) and Thailand (30\%) [14]. Intriguingly, cross-national colistin susceptibility stood at $98 \%$ on average at that time [14].

Applied to clinical aspects, secondary infection of snakebites often arises from mixed pathogen like other animal bites. In a 10-year-long Taiwanese cross-sectional study, $P$. aeruginosa was detected in $23.8 \%$ of patients with subsequent infections [15]. In fact, among all Gram-negative aerobes, $P$. aeruginosa was the second most prevalent that followed M. morganii and Enterococcus species [15].

At the time, there are no guidelines or consensus of antibiotics selection for snakebites [16]. Nonetheless, healthcare professionals should be alerted as MDR and colistin-resistant $P$. aeruginosa strains were recognized in our study. That is, in the context of snakebites in Taiwan, the victim is actually running a high risk of treatment failure.

On the other hand, adaptive colistin resistance of $P$. aeruginosa could also be induced by subinhibitory doses [17]. Surprisingly, the systemic review on pharmacokinetics and pharmacodynamics of colistin is inadequate, even after over-50-year-long clinical adoption [18]. It is foreseeable that colistin administration at a sublethal concentration might be insensible to clinicians and leads to adaptive resistance subsequently. Furthermore, stimulation from colistin at a sublethal 
level is likely to trigger resistance to aminoglycosides and quinolones [19].

Generated to $P$. aeruginosa of reptile origin, appalling colistin resistance at $55 \%$ probably results from exposure to unspecified antibacterial peptides [20]. Concerning serine- $\beta$ lactamases, $100 \%$ prevalence of the resistance gene blaTEM among $P$. aeruginosa within natural environments was once reported by Igbinosa et al. before [21], whereas strains extracted from human infection expressed blaTEM at a far below ratio [22]. Nonetheless one-tenth of isolates were still susceptible to cefotaxime and the discrepancy between genotypes and phenotypes probably indicated conditional expression of Ambler class A $\beta$-lactamases genes. In addition, our work highlighted high frequency of blaAmpC. Since several antibiotics could upregulate AmpC production [23], none of them were employed in our work in order to avoid batch errors. In other words, route of exposure prior to the recruitment might be the untold story. As all three Ambler classes of $\beta$-lactamases contributed to cefotaxime resistance, that raised a question about the hierarchy of single resistance gene. In contrast, it could also be possible for a universal cue to orchestrate expression of those genes, simultaneously.

Undoubtedly, certain limitations of our work exist and it takes further studies to discover the missing puzzle pieces. Since meropenem-resistant $P$. aeruginosa was detected, extensive work on genes encoding for carbapenemases such as OXA-48 and metallo- $\beta$-lactamases is required $[24,25]$. Similarly, polymyxin resistance genes, PA4773-PA4775, for example, require thorough investigation [26]. However, impact of resistance genes might be overestimated as they are not constitutive to gene expression. Meanwhile, susceptibility profiles of quinolones should be established in the near future as MDR and colistin resistance among $P$. aeruginosa from snakes' oral cavity was noted.

Of note, oral flora suggests the gut microbiota of the preys, to some extent, as they might defecate even when being consumed [27]. Indeed, any creatures that are exposed to animal performance enhancers (APEs) and in contact with snakes are skeptical culprits. Also, environmental dissemination and unreported preexposures should be taken into account. The authorities should be cautious of disorganized regulation and reporting system of off-label antibiotic administration, of which biochemical characteristics of APEs and other administration details remain unclear as only gross doses in the unit of kilograms and animal species are reported [28, 29]. Otherwise the rooting issue of disseminating antibiotics at a sublethal dose will continue to threaten public health.

\section{Conclusion}

MDR and colistin-resistant $P$. aeruginos $a$ were identified from the snakes' oral cavity in Taiwan for the first time, which indicated high risk of medical treatment failure in the context of snakebites and the crucial role of antibiotic stewardship to prevent adaptive resistance. On the other hand, wide prevalence of serine- $\beta$-lactamases was also disclosed through our work. Nonetheless the correlation between genotypes and phenotypes still requires further investigation.

\section{Additional Points}

Highlights. (1) Pseudomonas aeruginosa are not uncommon in snakes' oral cavity. (2) Multidrug resistant and colistinresistant $P$. aeruginosa from snakes' oral cavity were discovered. (3) The lowest resistance rates were in aztreonam, piperacillin/tazobactam, and amikacin. (4) 100\% of the isolates harbor resistance gene blaTEM and high frequency of blaAmpC among $P$. aeruginosa isolates.

\section{Conflicts of Interest}

The authors declare that they have no conflicts of interest.

\section{Authors' Contributions}

Po-Yu Liu wrote the manuscript and collected samples. Yan-Chiao Mao revised the manuscript. Ling-Ling Weng conducted the data analysis. Shu-Ying Tseng contributed to the microbiological analysis. Chou-Chen Huang and ChingChang Cheng collected samples of snakes from different vet hospitals. Kwong-Chung Tung revised the manuscript. All authors contributed to data analysis, drafting, and critically revising the paper, read and approved the final manuscript, and agreed to be accountable for all aspects of the work.

\section{Acknowledgments}

The authors would like to express their sincere appreciation to Dr. Robert Chan and Ms. Chin Chang-Chien for their support and assistance. This work was supported by grants from the Taichung Veterans General Hospital (103DHA0500493) Taichung, Taiwan.

\section{References}

[1] C. S. Cabassi, S. Taddei, S. Cavirani, M. C. Baroni, P. Sansoni, and A. A. Romani, "Broad-spectrum activity of a novel antibiotic peptide against multidrug-resistant veterinary isolates," Veterinary Journal, vol. 198, no. 2, pp. 534-537, 2013.

[2] A. E. Van den Bogaard and E. E. Stobberingh, "Epidemiology of resistance to antibiotics: links between animals and humans," International Journal of Antimicrobial Agents, vol. 14, no. 4, pp. 327-335, 2000.

[3] C. Alvarez-Ortega, I. Wiegand, J. Olivares, R. E. W. Hancock, and J. L. Martínez, "The intrinsic resistome of Pseudomonas aeruginosa to $\beta$-lactams., Virulence, vol. 2, no. 2, pp. 144-146, 2011.

[4] A. J. Morrison and R. P. Wenzel, "Epidemiology of infections due to Pseudomonas aeruginosa," Clinical Infectious Diseases, vol. 6, no. Supplement 3, pp. S627-S642, 1984.

[5] F. M. Abrahamian and E. J. C. Goldstein, "Microbiology of animal bite wound infections," Clinical Microbiology Reviews, vol. 24, no. 2, pp. 231-246, 2011.

[6] A. Wolfensberger, H. Sax, R. Weber, R. Zbinden, S. P. Kuster, and M. Hombach, "Change of antibiotic susceptibility testing guidelines from CLSI to EUCAST: Influence on cumulative hospital antibiograms," PLoS ONE, vol. 8, no. 11, Article ID e79130, 2013. 
[7] D. Lin, S. L. Foley, Y. Qi et al., "Characterization of antimicrobial resistance of Pseudomonas aeruginosa isolated from canine infections," Journal of Applied Microbiology, vol. 113, no. 1, pp. 16-23, 2012.

[8] K. Harada, S. Arima, A. Niina, Y. Kataoka, and T. Takahashi, "Characterization of Pseudomonas aeruginosa isolates from dogs and cats in Japan: current status of antimicrobial resistance and prevailing resistance mechanisms," Microbiology and Immunology, vol. 56, no. 2, pp. 123-127, 2012.

[9] C. C. Pye, A. Singh, and J. S. Weese, "Evaluation of the impact of tromethamine edetate disodium dihydrate on antimicrobial susceptibility of Pseudomonas aeruginosa in biofilm in vitro," Veterinary Dermatology, vol. 25, no. 2, pp. 120-e34, 2014.

[10] Y. Morita, J. Tomida, and Y. Kawamura, "Responses of Pseudomonas aeruginosa to antimicrobials," Frontiers in Microbiology, vol. 4, article 422, 2014.

[11] I.-L. Chen, C.-H. Lee, L.-H. Su, Y.-F. Tang, S.-J. Chang, and J.-W. Liu, "Antibiotic consumption and healthcare-associated infections caused by multidrug-resistant gram-negative bacilli at a large medical center in taiwan from 2002 to 2009: implicating the importance of antibiotic stewardship," PLOS ONE, vol. 8, no. 5, Article ID e65621, 2013.

[12] S.-S. Jean, W.-S. Lee, K.-W. Yu et al., "Rates of susceptibility of carbapenems, ceftobiprole, and colistin against clinically important bacteria collected from intensive care units in 2007: results from the surveillance of multicenter antimicrobial resistance in Taiwan (SMART)," Journal of Microbiology, Immunology and Infection, pp. 1-8, 2015.

[13] W.-T. Lo, W.-J. Lin, T.-S. Chiueh, S.-Y. Lee, C.-C. Wang, and J.-J. Lu, "Changing trends in antimicrobial resistance of major bacterial pathogens, 1985-2005: a study from a medical center in northern Taiwan," Journal of Microbiology, Immunology and Infection, vol. 44, no. 2, pp. 131-138, 2011.

[14] R. E. Mendes, M. Mendoza, K. K. Banga Singh et al., "Regional resistance surveillance program results for 12 Asia-Pacific nations (2011)," Antimicrobial Agents and Chemotherapy, vol. 57, no. 11, pp. 5721-5726, 2013.

[15] C.-M. Chen, K.-G. Wu, C.-J. Chen, and C.-M. Wang, "Bacterial infection in association with snakebite: a 10 -year experience in a northern Taiwan medical center," Journal of Microbiology, Immunology and Infection, vol. 44, no. 6, pp. 456-460, 2011.

[16] P.-Y. Liu, Z.-Y. Shi, C.-F. Lin et al., "Shewanella infection of snake bites: a twelve-year retrospective study," Clinics, vol. 67, no. 5, pp. 431-435, 2012.

[17] J. E. Moore and J. C. Rendall, "Comparison of susceptibility of cystic-fibrosis-related and non-cystic-fibrosis-related Pseudomonas aeruginosa to chlorine-based disinfecting solutions: Implications for infection prevention and ward disinfection," Journal of Medical Microbiology, vol. 63, no. 9, pp. 1214-1219, 2014.

[18] N. Martis, S. Leroy, and V. Blanc, "Colistin in multi-drug resistant Pseudomonas aeruginosa blood-stream infections: a narrative review for the clinician," Journal of Infection, vol. 69, no. 1, pp. 1-12, 2014.

[19] C. Muller, P. Plésiat, and K. Jeannot, "A two-component regulatory system interconnects resistance to polymyxins, aminoglycosides, fluoroquinolones, and $\beta$-lactams in Pseudomonas aeruginosa," Antimicrobial Agents and Chemotherapy, vol. 55, no. 3, pp. 1211-1221, 2011.

[20] L. Fernández, W. J. Gooderham, M. Bains, J. B. McPhee, I. Wiegand, and R. E. W. Hancock, "Adaptive resistance to the "last hope" antibiotics polymyxin B and colistin in Pseudomonas aeruginosa is mediated by the novel two-component regulatory system ParR-ParS," Antimicrobial Agents and Chemotherapy, vol. 54, no. 8, pp. 3372-3382, 2010.

[21] I. H. Igbinosa, U. U. Nwodo, A. Sosa, M. Tom, and A. I. Okoh, "Commensal Pseudomonas species isolated from wastewater and freshwater milieus in the Eastern Cape Province, South Africa, as reservoir of antibiotic resistant determinants," International Journal of Environmental Research and Public Health, vol. 9, no. 7, pp. 2537-2549, 2012.

[22] Z. A. Memish, A. Assiri, M. Almasri et al., "Molecular characterization of carbapenemase production among gram-negative bacteria in Saudi Arabia," Microbial Drug Resistance, vol. 21, no. 3, pp. 307-314, 2015.

[23] C. Juan, M. D. Maciá, O. Gutiérrez, C. Vidal, J. L. Pérez, and A. Oliver, "Molecular mechanisms of $\beta$-lactam resistance mediated by AmpC hyperproduction in Pseudomonas aeruginosa clinical strains," Antimicrobial Agents and Chemotherapy, vol. 49, no. 11, pp. 4733-4738, 2005.

[24] Y. Pfeifer, A. Cullik, and W. Witte, "Resistance to cephalosporins and carbapenems in Gram-negative bacterial pathogens," International Journal of Medical Microbiology, vol. 300, no. 6, pp. 371$379,2010$.

[25] S. Mohanty, V. Maurya, R. Gaind, and M. Deb, "Phenotypic characterization and colistin susceptibilities of carbapenemresistant of Pseudomonas aeruginosa and Acinetobacter spp.," Journal of Infection in Developing Countries, vol. 7, no. 11, pp. 880-887, 2013.

[26] S. Lewenza, "Extracellular DNA-induced antimicrobial peptide resistance mechanisms in Pseudomonas aeruginosa," Frontiers in Microbiology, vol. 4, 2013.

[27] R. L. Finley, P. Collignon, D. G. J. Larsson et al., "The scourge of antibiotic resistance: the important role of the environment," Clinical Infectious Diseases, vol. 57, no. 5, pp. 704-710, 2013.

[28] B. M. Marshall and S. B. Levy, "Food animals and antimicrobials: impacts on human health," Clinical Microbiology Reviews, vol. 24, no. 4, pp. 718-733, 2011.

[29] D. H. Lloyd, "Reservoirs of antimicrobial resistance in pet animals," Clinical Infectious Diseases, vol. 45, supplement 2, pp. S148-S152, 2007. 


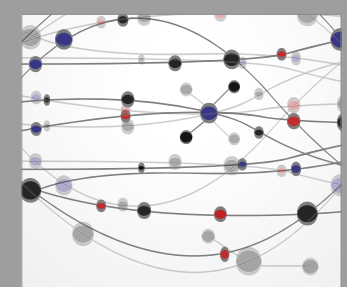

The Scientific World Journal
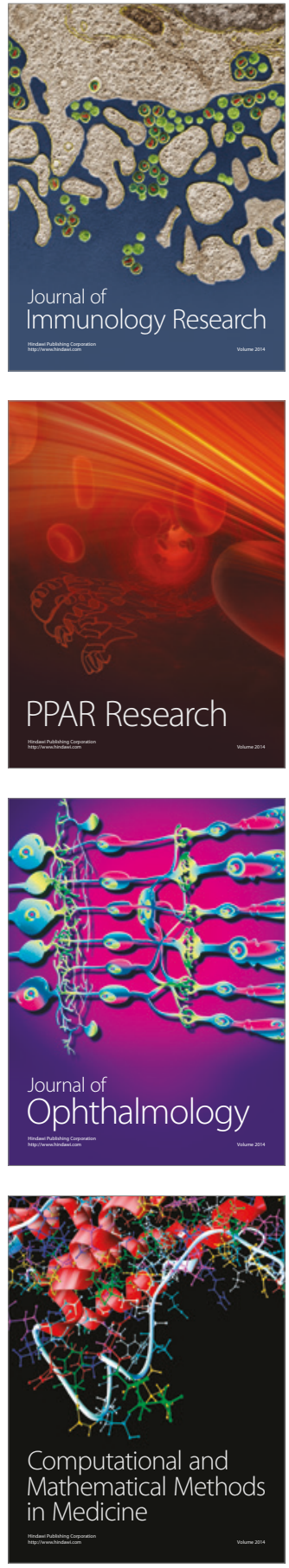

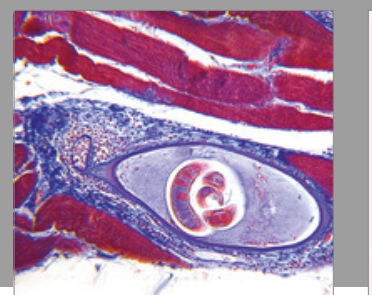

Gastroenterology Research and Practice
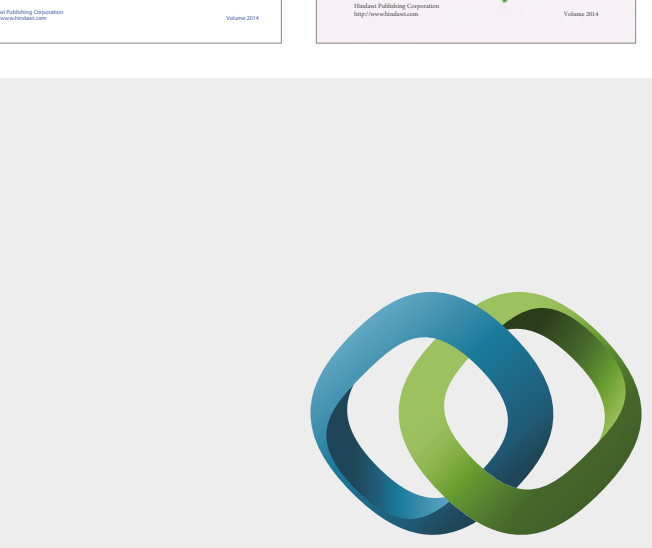

\section{Hindawi}

Submit your manuscripts at

https://www.hindawi.com
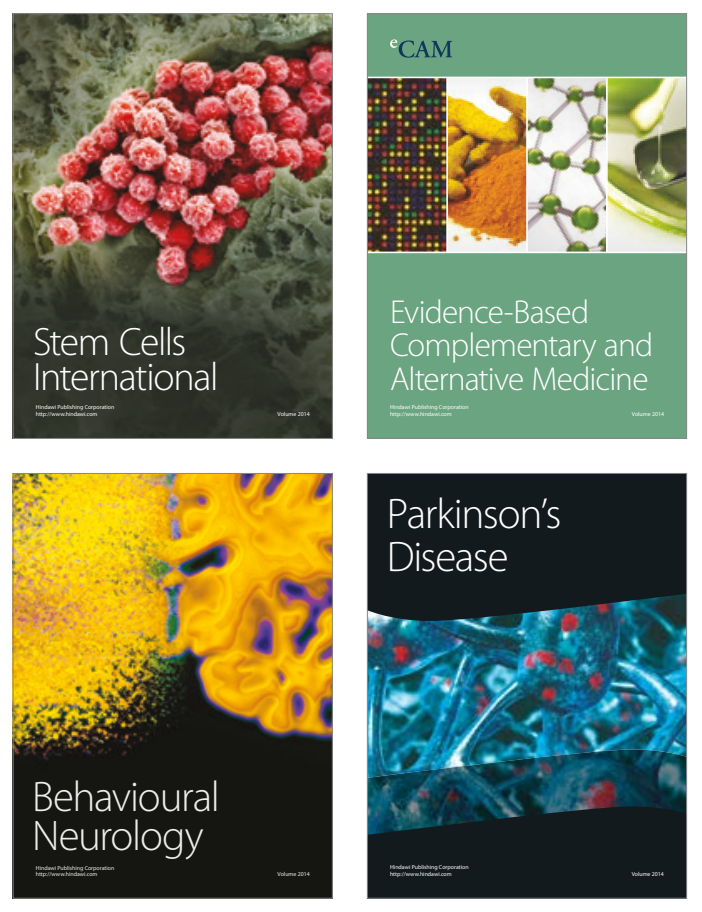
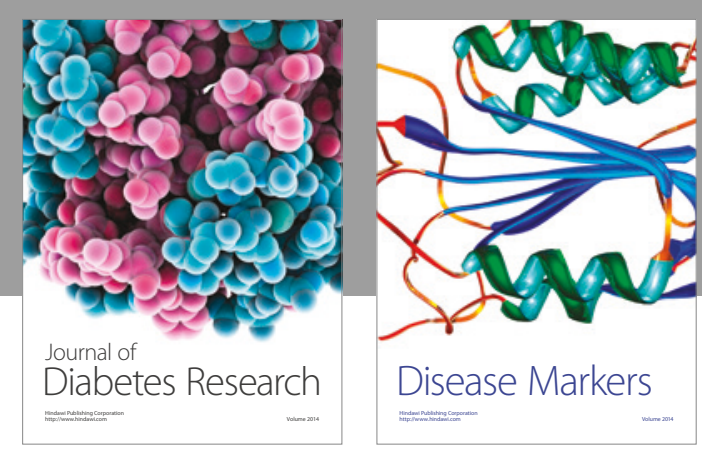

Disease Markers
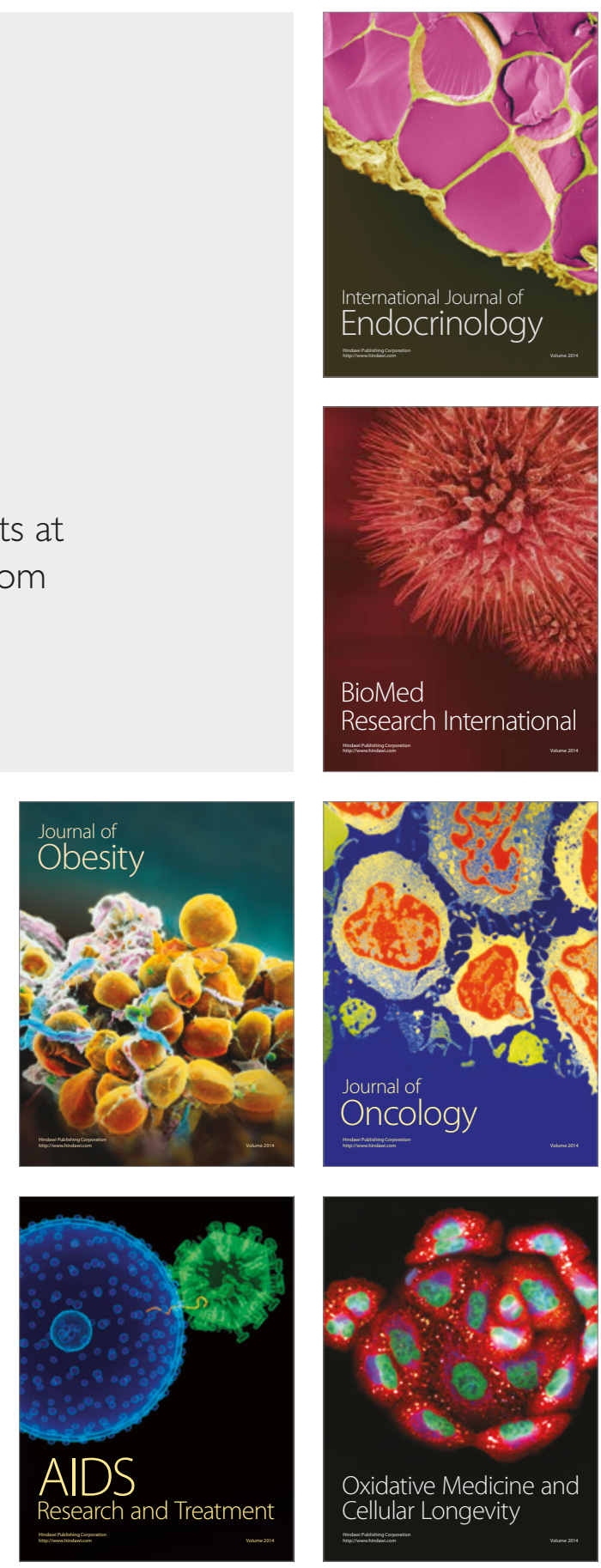